PALEO

Revue d'archéologie préhistorique

22 | 2011

Varia

\title{
Claude Barrière (1924-2011)
}

Michel Barbaza

\section{(2) OpenEdition}

Journals

Édition électronique

URL : http://journals.openedition.org/paleo/2057

DOI : $10.4000 /$ paleo.2057

ISSN : 2101-0420

Éditeur

SAMRA

Édition imprimée

Date de publication : 1 décembre 2011

Pagination : 14-18

ISSN : 1145-3370

\section{Référence électronique}

Michel Barbaza, «Claude Barrière (1924-2011) », PALEO [En ligne], 22 | 2011, mis en ligne le 13 avril

2012, consulté le 07 juillet 2020. URL : http://journals.openedition.org/paleo/2057 ; DOI : https://

doi.org/10.4000/paleo.2057

\section{(c) $(1)(9)$}

PALEO est mis à disposition selon les termes de la licence Creative Commons Attribution - Pas d'Utilisation Commerciale - Pas de Modification 4.0 International. 


\section{Claude BARRIÈRE (1924-2011)}

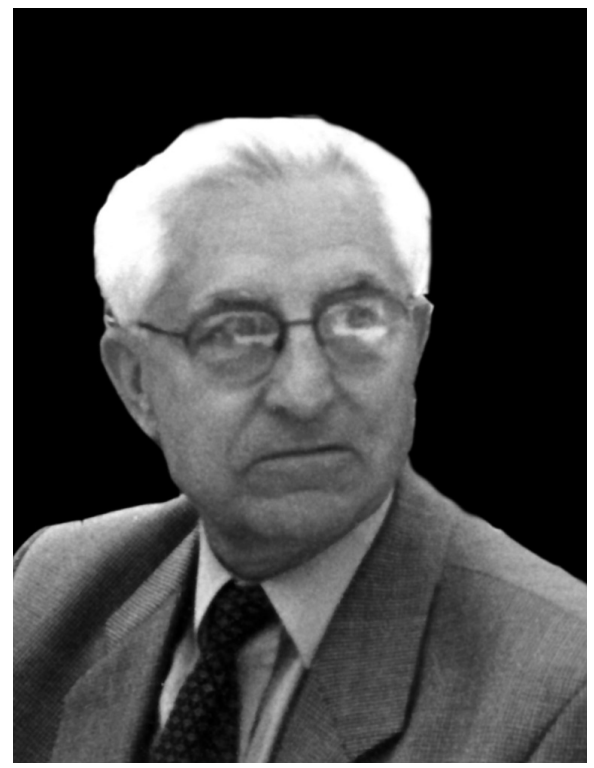

Claude Barrière, Professeur de Préhistoire à l'Université de Toulouse-Le Mirail, nous a quitté au terme d'une vie professionnelle dédiée à l'enseignement et à la recherche archéologique.

Né en 1924 à Périgueux, Claude Barrière, géographe de formation mène à partir de 1952 une carrière de professeur de collège puis de lycée, tout en consacrant ses loisirs et ses vacances à la recherche archéologique sur l'époque gallo-romaine et surtout sur la Préhistoire du nord de la Dordogne d'où il est originaire. En 1949, il fait la connaissance du Professeur LouisRené Nougier, premier et nouveau professeur de Préhistoire à la Faculté des Lettres de l'Université de Toulouse où il assure la relève des grands précurseurs que furent Emile Cartailhac et le Comte Henri Bégouën. Cette rencontre l'oriente définitivement vers l'étude des périodes anciennes de l'aventure humaine. Professeur au Lycée de Périgueux, il se désole des destructions archéologiques en cours et assure, de 1958 à 1970, des surveillances de récupération sur les chantiers de construction ainsi que des fouilles au cœur de la ville romaine de Vésonne.

Littéraire de formation, mais entraîné à la rigueur scientifique et géographique par les Professeurs P. Gourou, L. Papy, $\mathrm{H}$. Enjalbert et en géologie par le professeur E. Daguin ; plongé dans l'archéologie dès l'enfance par son père, Claude Barrière ne s'est jamais enfermé dans une étroite spécialisation et a maintenu cette disposition pour répondre aux exigences de l'enseignement. Son activité archéologique couvre de même un vaste champ chronologique.

En 1954, il soutient à Toulouse son Doctorat d'Etat en Archéologie préhistorique, le deuxième en France dans cette discipline, avec une thèse sur « Les civilisations tardenoisiennes en Europe occidentale ». Cet ouvrage fait le point sur les connaissances du moment relatives au Mésolithique qui comble « le hiatus » entre Paléolithique et Néolithique depuis longtemps établis. II fait table rase de théories sans fondements véritables et aide ainsi à une relance en Europe des études sur les cultures Epipaléo-mésolithiques. Sa thèse complémentaire sur "Les étapes du peuplement entre Dronne et Tardoire » apporte des connaissances nouvelles sur la Préhistoire du nord-ouest de la Dordogne, alors totalement négligée par les chercheurs, et y applique les méthodes d'analyse géographique préconisées par Louis-René Nougier dans sa propre thèse complémentaire.

En 1960, Claude Barrière est nommé Chef de Travaux à l'Institut d'Art Préhistorique de la Faculté des Lettres de Toulouse, renforçant ainsi une discipline de l'Enseignement supérieur français, pour longtemps encore mal dotée. En 1961, il est MaîtreAssistant. En 1978, il devient Maître de Conférences, puis Professeur en 1979. En 1980, il prend la Direction de l'Institut d'Art Préhistorique, au numéro 56 de la rue du Taur à Toulouse, et de sa publication "Travaux de l'Institut d'Art Préhistorique " créée en 1958. II y assurera son enseignement avant et après le déménagement des collections pédagogiques à la nouvelle Université du Mirail. Claude Barrière part à la retraite le 1er octobre 1989 , avec l'inquiétude de voir l'enseignement de l'art préhistorique disparaître à Toulouse. II réagit à cette situation en réalisant une exposition d'art préhistorique sur la base des recherches faites à l'Université de Toulouse. Une version abrégée, faite à la demande du Centre Georges-Pompidou de Beaubourg, voyagera dans toute la France. 
Soumis au rythme soutenu des avancées de la recherche archéologique et de la vie moderne en général, les générations actuelles et futures de jeunes préhistoriens retiendront certainement plusieurs points principaux de son œuvre : sa magnifique thèse d'Etat sur les civilisations tardenoisiennes, travail précurseur et véritablement novateur à son époque qui a donné aux ensembles du Mésolithique à armatures larges ou second Mésolithique ses lettres de noblesse, ses fouilles archéologiques sous le porche de Rouffignac qui ont contribué à la structuration chronologique et culturelle de l'ensemble du Mésolithique méridional, ses relevés systématiques du dispositif pariétal gravé de la grotte de Gargas. ainsi que ses travaux sur les parois ornées de la grotte de Rouffignac qui aboutissent, à partir de la structuration du Grand plafond, à une vision personnelle et originale de l'art de cette cavité. En point d'orgue, ses travaux aux grottes de Combarelles, couronnés par une monographie monumentale, apparaissent comme une magnifique conclusion de sa carrière. Les plus anciens, ou les plus attentifs d'entre nous, se souviendront néanmoins d'une œuvre personnelle beaucoup plus ouverte et éclectique comme le reflète la centaine de références sur ses travaux. II reviendra spontanément à l'esprit de ses proches et particulièrement de ses étudiants, la disponibilité du pédagogue, l'étendue de son érudition et, plus généralement, la très belle humanité de son comportement à l'égard de chacun.

Dans son quotidien d'universitaire, Claude Barrière a toujours été très attentif au sort de ses proches, collègues et collaborateurs, étudiants de premières années ou doctorants, à tous ceux pour lesquels sa responsabilité, d'une manière ou d'une autre, était engagée, afin qu'ils puissent assurer leurs tâches dans les meilleures conditions de responsabilité et de liberté possibles. Retiré par choix personnel de la recherche en Préhistoire, ne doutons pas qu'il aurait été fier du développement de cette activité à l'Université de Toulouse qui abrite désormais une des plus grandes et actives équipes de préhistoriens de France, lui qui fit de la primauté toulousaine, peu d'entre nous le savent, le grand combat de sa vie professionnelle. Par leur réussite, les jeunes générations lui rendent un hommage constant.

\section{Michel Barbaza}

\section{Principaux travaux et publications}

\section{7}

Habitats néolithiques dans la région de Piégut-Pluviers (Dordogne). Gallia t. V, 2, p. 405-17.

Le site archéologique de Ste Radegonde (Baignes, Charente). Gallia t.V, 2, p. 454-59.

\section{8}

Un habitat néolithique fossilisé en Périgord : le Pays de Piégut. Annales du Midi, t. LXI.

\section{9}

Le Piégutais : l'occupation du sol. Revue de Géographie des Pyrénées \& du Sud-Ouest, t. XX, 1-2.

\section{0}

Un village protohistorique : Puygaud (Dordogne). Congrès Préhistorique de France. Paris, 1950.

\section{1}

Un exemple de permanence de l'habitat : Puydivers (Dordogne). B.S.P.F., t. XLVIII, 1.

L'oppidum de Sendougne (Dordogne). B.S.P.F., t. XLVIII, 7.

1952

Note pour la connaissance de la Double préhistorique. B.S.P.F., t. XLIX, 7, p.341-349.

Réponse à la note de $M$. Gaillard sur les pierres à fusil. B.S.P.F., t. XLIX, p. 99-100.

Note sur un microburin magdalénien à la Souquette de Sergeac. B.S.P.F. t. XLIX, 7, p.573-574.
L'oppidum de La Boissière dit "Camp de César» à Périgueux. B.S.H.A.P., Périgord, t. LXXIX.

\section{3}

Le champ du Chalard ; le Bois de Sérillac ; l'enceinte de Lastours. B.S.H.A.P., Périgord, t. LXXX.

La métallurgie protohistorique et gallo-romaine en Nontronnais, B.S.H.A.P., Périgord, t. LXXX.

Le Champ du Pouyoulet. B.S.H.A.P. Périgord, t. LXXX.

Bordeaux dans la toponymie et la topographie aquitaine. Revue d'Histoire de Bordeaux et de la Gironde. (En collaboration avec $\mathrm{P}$. Barrière).

Le camp vitrifié de Castelsarrazi n'existe pas. C.P.F., Strasbourg 1953, p. 107-114.

Meule préhistorique complète du Roc Alan (Sauveterre-laLemance). C.P.F., Strasbourg 1953. p. 114-117.

Le gisement néolithique post-campignien de Ségor (Charente-Maritime). C.P.F., Strasbourg 1953, p. 96-106.

\section{4}

Les Civilisations tardenoisiennes en Europe occidentale. Ed. Bière, p. 340, 6 cartes, 135 fig.

Les étapes du peuplement dans les pays entre Dronne et Tardoire. Imp. C.R.D.P., Bordeaux, 250 p., 10 cartes, 5 pl., 86 figures.

Les origines du Tardenoisien. C.I. Anth. et Arch. Madrid.

1955

Les genres de vie Tardenoisiens, I.G, 19, $\mathrm{n}^{\circ} 3$. 
Les civilisations tardenoisiennes en Europe occidentale. Pallas IV.

L'oppidum de Sendougne (Dordogne), C.P.F. Poitiers, 1956, p.219.

\section{6}

Le Camp de César de la Bessède. B.S.H.A.P., Périgord.

Le peuplement préhistorique des environs de Belvès (Dordogne). C.P.F. Poitiers 1956. p.234-42.

Géographie humaine : quelques exemples de peuplement préhistorique. Acta Geographica.

\section{9}

Premiers aperçus sur la céramique de Rouffignac. Pallas VIII, 1958.

\section{Editorial. Cahiers d'Archéologie du Nord-Est.}

Les fouilles de Rouffignac. Cahiers d'Archéologie du NordEst.

Les fouilles de Rouffignac : découverte de 6 squelettes tardenoisiens. Pallas VIII, p. 3-8.

Le gisement de de St Laurent la Vallée (Dordogne) (résumé). C.P.F., Monaco 1959.

Deux « pierres figures ». Bull. Soc. des Eyzies.1956, n 6.

Un anthropomorphe en os de Pair-Non-pair (Gironde), Bull. Soc. des Eyzies, 1956.

Les civilisations tardenoisiennes en Europe occidentale. B.S.P.F., t. LII, 1955, p.204-206.

\section{0}

L'origine du Tardenoisien. Caesaraugusta 11-12, 1958, p. 7-13.

Un petit trésor monétaire dans la grotte de Rouffignac. Travaux Institut d'Art Préhistorique, Toulouse.

\section{1}

Un sépulture du Premier Age du Fer à Plazac (Dordogne). Travaux Institut d'Art Préhistorique, Toulouse, p. 19-28.

Le gisement solutréen de St Laurent la Vallée (Dordogne). Travaux Institut d'Art Préhistorique, Toulouse, p. 29-68.

\section{2}

Le gisement de Rouffignac. Vues d'ensemble des résultats des fouilles 1957-62. C.I. Anthrop. Préhist. Rome.

Deux oeuvres d'art sauveterriennes à Rouffignac. Travaux Institut d'Art Préhistorique, Toulouse, p. 20.

L'utilisation des reliefs rocheux dans l'art paléolithique supérieur : deux exemples nouveaux à Gargas et au Portel. Travaux Institut d'Art Préhistorique, Toulouse V , p. 21-22.
Les gravures de la Grotte du Gazel à Sallèles-Cabardès (Aude). Travaux Institut d'Art Préhistorique, Toulouse, pp.23-32.

\section{3}

Les peintures du second étage de, Rouffignac et la signification de l'art préhistorique. Hommage à BoschGimpera, Mexico 1963, p. 345-351 (en collaboration avec L.R. Nougier).

Conférence sur le Tardenoisien (publication de la Soc. Royale d'Anthrop. de Bruxelles).

\section{4}

Les empreintes humaines de Lascaux. Hommage à l'abbé Breuil, Barcelone 1964, p. 80.

\section{5}

Flore et faune de Rouffignac. Travaux Institut d'Art Préhistorique, VII, Toulouse, 1967, p.7-12.

La coupe actuelle du gisement du château des Eyzies. Travaux Institut d'Art Préhistorique, VII, Toulouse, 1967, pp. 13-18.

Gravures schématiques en Dordogne. Travaux Institut d'Art Préhistorique, VII,Toulouse, p. 19-20 (en collaboration avec L.R. Nougier).

La nbuvelle grotte des Fieux et ses mains négatives. L.R.N.C.B. Science Progrès, La Nature, mars 1965.

Les couteaux-faucilles de Rouffignac. Hommage à l'abbé Breuil, Lisbonne 1965, pp. 73-78.

La grotte ornée des Fieux. Travaux Institut d'Art Préhistorique, VII, Toulouse, pp. 115-134 (avec L.-R. Nougier).

\section{6}

L'art paléolithique supérieur occidental. Travaux Institut d'Art Préhistorique, Toulouse Travaux I.A.P. Toulouse VIII-IX, 196667, pp. 151-71.

\section{8}

Les gravures de la Grotte de la Mairie à Teyjat (Dordogne). Travaux Institut d'Art Préhistorique, X, Toulouse, p. 1-13.

La grotte de Gahuzère I à Montmaurin, Haute Garonne. Travaux Institut d'Art Préhistorique, X,Toulouse, p.14-25.

A propos des industries en quartzite de la Garonne. Travaux Institut d'Art Préhistorique, X,Toulouse, p.26-30.

\section{0}

Les techniques de la gravure à Rouffignac. Travaux Institut d'Art Préhistorique, XII,Toulouse, p. 4-1,04.

Trouvailles paléontologiques à Montmaurin. Travaux Institut d'Art Préhistorique, Toulouse, XII, p. 105-130.

Scène anthropomorphique à Font de Gaume. Bul. Soc. Préh. Ariège, t. XXIV. 
1969

Epipaléolithique-Mésolithique. Les microlithes géométriques. B.S.P.F., t. 66, 1969, pp. 355-366. (en participation au Groupe d'Etude de l'Epipaléolithique-Mésolithique)

\section{0}

Catalogue illustré de pièces préhistoriques inédites du Grand-Pressigny. Bull. des Amis du Musée du Grand Pressigny.

1972

Epipaléolithique-Mésolithique. Les armatures non géométriques B.S.P.F., t.69, p. 364-375. (en collaboration avec les membres du G.E.E.M.).

Grotte de la Mairie, Teyjat. Une nouvelle petite gravure. Travaux Institut d'Art Préhistorique, XIV, Toulouse.

A propos de la sidérurgie gallo-romaine en Périgord. Bonner Hefte zur Vorgeschichte, 3, Bonn, p. 3-11.

Les gravures de Gargas. Congrès des Soc. Savantes. Montauban.

1973

Rouffignac. Son archéologie. Travaux Institut d'Art Préhistorique, XV, 1, Toulouse, p. 160.

Le gisement de Merles (Tarn XV 1973, pp.1.6l-211. Mémoires de l'Institut d'Art Préhistorique de Toulouse, et Garonne). Travaux Institut d'Art Préhistorique, XV, Toulouse. (en collaboration avecc $\mathrm{Cl}$. et $\mathrm{A}$. Lagarrigue)

Les gravures en tracé digital de-la Grotte de Gargas. Bul. Soc. Préh. Ariège, XXVIII,

Rouffignac. Son archéologie. Travaux Institut d'Art Préhistorique, XV, 2, Toulouse.

1974

Documents céramiques pyrénéens. Travaux Institut d'Art Préhistorique, XVI, Toulouse.

Un hachereau moustérien du Périgord. Travaux Institut d'Art Préhistorique, XVI, Toulouse.

1975

Rouffignac. Son archéologie. Travaux Institut d'Art Préhistorique, XVII, 3, Toulouse.

- La grotte du Cluzel de Camboulives. Travaux Institut d'Art Préhistorique, VII, Toulouse.

La grotte de Gargas. Bul. A.F.E.Q., 3-4, p. 201-203.

1976

Une exploitation d'argile gallo-romaine dans la grotte de Rouffignac. Travaux Institut d'Art Préhistorique, XVIII.

Un nouveau racloir triangulaire en bronze, Travaux Institut d'Art Préhistorique, XVIII, Toulouse.
Un vase inédit du Truc de Bourdieu, Mios. Travaux Institut d'Art Préhistorique, Toulouse Travaux I.A.P. Toulouse XVIII, 1976,2p.

Une pointe moustérienne particulière. Travaux Institut d'Art Préhistorique, XVIII, Toulouse.

Note complémentaire au sujet de St Germain la Rivière. Travaux Institut d'Art Préhistorique, XVIII, Toulouse.

L'évoution de l'art pariétal de Gargas. B.A.R. suppléments Oxford, $410 \mathrm{p}$.

L'évolution de l'art pariétal de Gargas. I $X^{e}$ Congres U.I.S.P.P. Nice, 1976.

L'outillage du fonds commun. B.S.P.F., p. 319-332 (en collaboration avec les membres du G.E.E.M.).

\section{7}

Le gisement Moulin de Vent-Artenac des Sablières de Bonnes, Charente. Travaux Institut d'Art Préhistorique, XIX, Toulouse.

Notes complémentaires à l'archéologie de Rouffignac. Travaux Institut d’Art Préhistorique, XIX, Toulouse.

Dépôt CU à Merles (Tarn et Garonne). Travaux Institut d'Art Préhistorique, XIX,Toulouse.

A propos des mains de Gargas. B.S.P.F. C.R.S.M. du 8 novembre 1977, p. 226-228.

L'abbè Breuil dans la région toulousaine. Bul. S.M.S.P., p.13-26.

Anneaux-monnaies celtiques. Travaux Institut d'Art Préhistorique XX, , Toulouse, p. 1-10.

\section{9}

Prospections archéologiques aux confins occidentaux du Périgord. Travaux Institut d'Art Préhistorique, XX, p. 1-66.

\section{0}

La grotte des Combarelles: quelques gravures nouvelles. Travaux Institut d'Art Préhistorique, XXII, Toulouse, p. 137-148

Le grand plafond de Rouffignac. L'organisation. B.S.P.F., t. 77 , p. $269-276$.

\section{1}

La Font-Bargeix. Aménagements médiévaux. Travaux Institut d'Art Préhistorique, XXIII, Toulouse.

La grotte des Combarelles. Relevés des gravures pariétales de la paroi. Travaux Institut d'Art Préhistorique, XXIII, Toulouse, p. 13-96.

Les associations de figures dans l'art pariétal de Rouffignac. Bul. Soc. Et. Eyzies, 30.

\section{2}

Rouffignac : I'organisation pariétale. Xème Congrès U.I.S.P.P. Mexico. 


\section{3}

Les gravures pariétales de Combarelles I, paroi gauche. Travaux Institut d'Art Préhistorique, XXV, Toulouse.

L'art pariétal de Rouffignac. Fondation Singer-Polignac, Picard éd., Paris. 250 p. 500 figures, 8 pl. couleur.

\section{3}

Combarelles I, les peintures pariétales de la galerie antérieure. Travaux Institut d'Art Préhistorique, XXV.

Observations de typologie fonctionnelle sur l'outillage magdalénien de la Font-Bargeix, Dordogne. Travaux Institut d'Art Préhistorique, XXV,Toulouse.

Le thème du serpent à Rouffignac. Prehistoric Art and Religion. Valcamonica Symposium, 1979, p. 323-330,

A l'école des mammouths de Rouffignac. Homenaje al Prof. Martin Almagro Basch. Madrid, pp. 281-286.

\section{4}

La Fongt-Bargeix. Première présentation des fouilles. Travaux Institut d'Art Préhistorique, XXVI , p. 15-40.

La grotte Sindou, Sénaillac-Lauzès» Lot. Travaux Institut d'Art Préhistorique, XXVI, Toulouse, p. 41-64. (En collaboration avec François Briois).

\section{5}

Les gravures pariétales de Combarelles I, la galerie profbnde. Travaux Institut d'Art Préhistorique, XXVII, p. 6.

\section{6}

Les figures animales transformées de Combarelles I. Estudios en Homenage al Prof. A. Beltran Martinez. Universit. Saragosse, 1986, pp. 85-91.

A propos d'Adam et Eve de Rouffignac. Travaux Institut d'Art Préhistorique, XXVIII, Toulouse, p. 57-58.

Lexique d'art préhistorique. Travaux Institut d'Art Préhistorique, XXVIII, Toulouse, p.163-208.

\section{7}

Les dessins de la galerie profonde de Combarelles I. Travaux Institut d'Art Préhistorique, XXIX, p. 81.
La Font-Bargeix (Champeau, Dordogne). Le niveau médiéval. Travaux Institut d'Art Préhistorique, XXIX,Toulous, p. 33-54.

Premières données sur l'art pariétal du Ker de Massat. Xlème Congrès International, Mayence sept. 1987.

La grotte du Ker de Massat, Ariège. Quelques aspects nouveaux des gravures. Ars Praehistorica vol. 5-6, Madrid, p. 59-69.

\section{8}

La Font Bargeix. Les niveaux magdaléniens. I. Travaux Institut d'Art Préhistorique, XXX,Toulouse, pp. 39-109.

\section{9}

La Font Bargeix. Champeau La Chapelle Pommier Dordogne : outillage, faune, matériaux, II, Travaux Institut d'Art Préhistorique, XXXI, Toulouse, p. 39-144.

CR. Leroi-Gourhan. A. Travaux Institut d'Art Préhistorique, XXXI, Toulouse.

\section{0}

L'art pariétal du Ker de Massat. Presses Universitaires du Mirail. 144p., I37 fig. 139 photo.

La Font Bargeix. Les niveaux magdaléniens, III. Travaux Institut d'Art Préhistorique, XXXII,Toulouse.

La Font-Bargeix. Art et archéologie. Colloque du cinquantenaire de Lascaux. Montignac. 10-14 septembre 1990.

La Font-Bargeix. L'art pariétal. Travaux Institut d'Art Préhistorique, XXXII, Toulouse (en collaboration avec G. et B. Delluc).

\section{7}

L'art pariétal des grottes des Combarelles. $1^{\text {er }}$ supplément à Paléo. 609 p. 60 fig. 60 pl. h.t. 